Pacific Journal of Mathematics

ON SPLITTING IN HEREDITARY TORSION THEORIES 


\section{ON SPLITTING IN HEREDITARY TORSION THEORIES}

\section{ROBERT L. BERNHARDT}

Let $(\mathscr{T}, \mathscr{F})$ denote a hereditary torsion theory for the category of modules over a ring $R$. In this paper the splitting of projective modules is studied, and it is shown that this is not equivalent to the splitting of quasi-projective modules. In addition, situations arising from the class of torsion modules $\mathscr{T}$ (or the class of torsionfree modules $\mathscr{F}$ ) being contained in the injective or in the projective modules are considered, and several conditions sufficient for an especially strong form of splitting are given. Finally when $\mathscr{T}$ is closed under injective envelopes the following is shown: every module splits if $R$ is an artinian generalized uniserial ring, and projective modules split if $R$ is a $Q F-2$ ring.

The term "ring" will mean an associative ring with unity 1 , and all modules are assumed to be unitary left modules. We denote the category of all modules over a ring $R$ by ${ }_{R} \mathscr{K}$. Dickson [6] defined a torsion theory for ${ }_{R} \mathscr{C l}$ to be a pair $(\mathscr{T}, \mathscr{F})$ of classes of modules satisfying the following:

(a) $\mathscr{T} \cap \mathscr{F}=0$;

(b) $\mathscr{T}$ is closed under homomorphic images and $\mathscr{F}$ is closed under submodules;

(c) For each module $M$ there exists a (unique) submodule $M_{t} \in$ $\mathscr{T}$ such that $M / M_{t} \in \mathscr{F}$.

A torsion theory $(\mathscr{T}, \mathscr{F})$ is said to be hereditary if $\mathscr{T}$ is closed under submodules, and stable if $\mathscr{T}$ is closed under injective envelopes. We remark that from (b) above it is clear that $\operatorname{Hom}(T, F)=0$ for all $T \in \mathscr{T}$ and all $F \in \mathscr{F}$; also Dickson has shown that $\mathscr{T}$ is closed under submodules if and onlf if $\mathscr{F}$ is closed under injective envelopes. In this paper we shall always be concerned with hereditary torsion theories.

If $\mathscr{T}$ is a hereditary torsion class, then Gabriel [8] has shown that $\mathscr{T}$ is uniquely associated with an (topologizing and) idempotent filter

$F(\mathscr{T})=\{L \leqq R \mid L$ is a left ideal of $R$ and $R / L \in \mathscr{T}\}$. Moreover, $\mathscr{T}$ is a torsionfree class for some torsion class $\mathscr{C}$ if and only if $F(\mathscr{T})$ contains a unique minimal left ideal (see [9]); in this case Jans has called $\mathscr{T}$ a torsion-torsionfree (TTF) class, and we shall call $(\mathscr{T}, \mathscr{F})$ and $(\mathscr{C}, \mathscr{T})$ the torsion theories associated with $\mathscr{T}$. If $R$ is a right perfect ring, Alin [1] has shown that every hereditary torsion class for ${ }_{R} \mathscr{C l}$ is a TTF class. 
If $(\mathscr{T}, \mathscr{F})$ is a hereditary torsion theory for ${ }_{R} \mathscr{C l}$ and if $M \in$ ${ }_{R} \mathscr{L}$, we say that $M$ splits provided $M=M_{t} \oplus M^{\prime}$; we shall call $(\mathscr{T}, \mathscr{F})$ splitting if every module in ${ }_{R} \mathscr{C l}$ splits. We say that $(\mathscr{T}, \mathscr{F})$ is centrally splitting provided $\mathscr{T}$ is a TTF class with associated torsion theories $(\mathscr{T}, \mathscr{F})$ and $(\mathscr{C}, \mathscr{T})$, and $M=M_{t} \oplus M_{c}$ (i.e., $M$ is the direct sum of its two torsion submodules) for every $M \in_{R} \mathscr{M}$. Centrally splitting is clearly a strong form of splitting; the interested reader may see [5] for more information on this topic.

1. Splitting in projective modules. In this section we shall study the dual for projective modules to the following result of Armendariz [3] on the splitting of injective modules. We denote the injective envelope of a module $M$ by $E(M)$.

THEOREM A (Armendariz). If $(\mathscr{T}, \mathscr{F})$ is a hereditary torsion theory, then the following are equivalent:

(1) $\mathscr{T}$ is stable;

(2) Every injective module splits;

(3) Every quasi-injective module splits;

(4) $E\left(M_{t}\right)=E(M)_{t}$ for every $M \in_{R} \mathscr{C l}$.

If $N$ is a submodule of the module $M$, we call $N$ invariant in $M$ provided that $f(N) \subseteq N$ for every endomorphism $f$ of $M$. We call $N$ small in $M$ provided that if $K$ is a submodule of $M$ and if $K+N$ $=M$, then $K=M$. We shall say that a class $\mathscr{C}$ of modules is closed under projective covers provided that whenever $M \in \mathscr{C}$ has a projective cover $P(M)$, then $P(M) \in \mathscr{C}$.

Theorem 1.1. Let $(\mathscr{T}, \mathscr{F})$ be a hereditary torsion theory for ${ }_{R} \mathscr{C l}$ such that every torsionfree module has a projective cover. Then the following are equivalent:

(1) $\mathscr{F}$ is closed under projective covers;

(2) Every projective module splits.

Proof. (1) $\rightarrow(2): \quad$ Let $Q$ be a projective module, and let $\pi: P\left(Q / Q_{t}\right)$ $\rightarrow Q / Q_{t}$ be the projective cover of $Q / Q_{t}$. Let $n$ be the natural epimorphism of $Q$ onto $Q / Q_{t}$. By [4, Lemma 2.3] there exists a monomorphism $h: P\left(Q / Q_{t}\right) \rightarrow Q$ such that $n h=\pi$ and such that $Q=\operatorname{Im} h+Q^{\prime}$, where $Q^{\prime} \subseteq \operatorname{Ker} n=Q_{t}$. But $\operatorname{Imh}$ is torsionfree; so that $\operatorname{Imh} \cap Q_{t}=0$ and $Q=\operatorname{Imh} \oplus Q_{t}$.

$(2) \rightarrow(1):$ Choose $M \in \mathscr{F}$, and let $\pi: P(M) \rightarrow M$ be the projective cover of $M$. Then $P(M)_{t} \subseteq \operatorname{Ker} \pi$, so that $P(M)_{t}$ is small in $P(M)$. But $P(M)$ splits by hypothesis; thus $P(M) \in \mathscr{F}$. 
EXAMPLE 1.2. The splitting of projective modules does not imply the splitting of quasi-projective modules in left artinian generalized uniserial rings.

Let $K$ be a field, and let $R$ be the ring of $4 \times 4$ upper triangular matrices over $K$. Let

$$
I=\left\{\left|\begin{array}{llll}
a_{11} & a_{12} & a_{13} & a_{14} \\
0 & 0 & a_{23} & a_{24} \\
0 & 0 & a_{33} & a_{34} \\
0 & 0 & 0 & 0
\end{array}\right| \mid a_{i j} \in K\right\} ;
$$

then $I$ is an idempotent, two-sided ideal of $R$. Thus by a result of Jans [9], $\mathscr{T}=\left\{M \in_{R} \mathscr{C l} \mid I M=0\right\}$ is a TTF class. Further $R \in \mathscr{F}$, so that every free module is torsionfree. Hence every projective module is torsionfree, and thus splits. Now let $e_{i j}$ denote the matrix with 1 in the $i^{\text {th }}$ row and $j^{\text {th }}$ column and 0 elsewhere, and let $J=R e_{14}$. Then $J$ is a two-sided ideal of $R$, and hence $M=R e_{e_{4}} / J e_{44}=R e_{44} / J$ is quasiprojective and indecomposable. But $M \notin \mathscr{T}$, and $R e_{24} / J \subseteq M_{t}$. Thus $M_{t}$ is a nontrivial submodule of $M$.

We next turn our attention to the quasi-projective cover; this was introduced in [12], and there it was shown that a sufficient (but not necessary) condition for the quasi-projective cover of a module $M$ to exist is that the projective cover of $M$ exist.

Proposition 1.3. Let $M$ be a quasi-projective module which has a projective cover. If $N$ is an invariant submodule of $M$, then the module $M / N$ is quasi-projective.

Proof. Let $\pi: P(M) \rightarrow M$ be the projective cover of $M$, and choose an endomorphism $f$ of $P(M)$. By [12, Proposition 2.2], $f$ induces an endomorphism $g$ of $M$ such that $g \pi=\pi f$. Let $K=\pi^{-1}(N)$; then $\pi f(K)=g \pi(K)=g(N) \subseteq N$, and hence $f(K) \subset \pi^{-1}(N)=K$. We have shown that $K$ is invariant in $P(M)$; thus by [12, Proposition 2.1] we have $P(M) / K \cong M / N$ is quasi-projective.

THeOREM 1.4. Let $M$ be a module with a projective cover, let $\pi^{\prime}: Q P(M) \rightarrow M$ denote the quasi-projective cover of $M$, and let $(\mathscr{T}, \mathscr{F}$ ) be a hereditary torsion theory for ${ }_{R} \mathscr{M}$. If $M \in \mathscr{F}$, then $Q P(M) \in \mathscr{F}$.

Proof. Let $\pi: P(M) \rightarrow M$ denote the projective cover of $M$; by [12, Propositions 2.6, 2.1 and 2.2] we have that $Q P(M) \cong P(M) / X$, where $X$ is the unique maximal invariant submodule of $P(M)$ contained in $\operatorname{Ker} \pi$. Let $n$ denote the natural epimorphism of $P(M)$ onto 
$Q P(M)$. Since $\operatorname{Ker} n \subseteq \operatorname{Ker} \pi$, we have that $\operatorname{Ker} n$ is small in $P(M)$, and thus $n: P(M) \rightarrow Q P(M)$ is the projective cover of $Q P(M)$. Further $Q P(M)_{t} \subseteq \operatorname{Ker} \pi^{\prime}$ since $M \in \mathscr{F}$, and also $Q P(M)_{t}$ is invariant in $Q P(M)$. Hence $Q P(M) / Q P(M)_{t}$ is quasi-projective by Proposition 1.3; thus $Q P(M)_{t}$ $=0$ by condition (3) of the definition of the quasi-projective cover in [12].

2. Classes of projective and injective modules. Let $(\mathscr{T}, \mathscr{F})$ be a hereditary torsion theory for ${ }_{R} \mathscr{C}$. In this section we investigate the following condition:

$\mathscr{T}$ is stable and all torsionfree modules are injective. This has been studied previously in [3] (also see [2] for the special case that $\mathscr{T}$ is the Goldie torsion class), where it was shown to imply that $(\mathscr{T}, \mathscr{F})$ is splitting. In Theorem 2.2 we shall give a statement equivalent to this condition, and, in addition, we shall show that it implies the much stronger result: $(\mathscr{T}, \mathscr{F})$ is centrally splitting. Finally we shall obtain a dual to Theorem 2.2.

Lemma 2.1. Let $(\mathscr{T}, \mathscr{F})$ be a hereditary torsion theory for ${ }_{R} \mathscr{M l}$, let $R=R_{t} \oplus K$, and let $\mathscr{F}$ be closed under homomorphic images. Then $R=R_{t}+K$ (ring direct sum), $\mathscr{T}$ is a TTF class, and $(\mathscr{T}, \mathscr{F}$ ) is centrally splitting.

Proof. Since right multiplication by an element of $R$ is a left $R$-homomorphism on $K$, and since $\mathscr{F}$ is closed under homomorphic images, $K$ is a two-sided ideal of $R$ and $R=R_{t}+K$.

By [5, Theorem 1] it now suffices to see that $\mathscr{T}$ is a TTF class. Choose $L \in F(\mathscr{T})$; then $K \cap L \in F(\mathscr{T})$, and hence $R / K \cap L \in \mathscr{T}$. Thus $K / K \cap L \in \mathscr{T}$. But $K \rightarrow K / K \cap L \rightarrow 0$ is exact and $K \in \mathscr{F}$; thus $K / K$ $\cap L \in \mathscr{T} \cap \mathscr{F}=0$ and $K=K \cap L \leqq L$. We have shown that $K$ is the unique minimal ideal in $F(\mathscr{T})$; thus $\mathscr{T}$ is a TTF class.

THEOREM 2.2. If $(\mathscr{T}, \mathscr{F})$ is a hereditary torsion theory for ${ }_{R} \mathbb{l l}$, then the following are equivalent:

(1) $\mathscr{T}$ is stable, and all torsionfree modules are injective;

(2) $\mathscr{F}$ is closed under homomorphic images, and all torsionfree modules are projective;

(3) $\mathscr{F}$ is clossed under homomorphic images, $R=R_{t}+K$ (ring direct sum), and $K$ is a semi-simple ring with minimum condition.

In addition, whenever (1), (2), and (3) are true, then $\mathscr{T}$ is a TTF class and $(\mathscr{T}, \mathscr{F})$ is centrally spilitting.

Proof. (1) $\rightarrow$ (3) follows from [3, Theorem 3.2].

$(3) \rightarrow(2)$ : If $M$ is a torsionfree module, then $R_{t} M=0$ and hence 
$M$ is a projective $K$-module. But now $M$ is a direct summand of a free $K$-module, and hence $M$ is a direct summand of a free $R$-module. Thus $M$ is projective as an $R$-module.

$(2) \rightarrow(1)$ : Choose $M \in \mathscr{T}$, and let $n$ be the natural epimorphism of $E(M)$ onto $E(M) / E(M)_{t}$. Since this torsionfree module is projective, there exists a monomorphism $f$ from $E(M) / E(M)_{t}$ into $E(M)$ such that $E(M)=\operatorname{Ker} n \oplus \operatorname{Im} f$. But $M \subseteq \operatorname{Ker} n$ and $M$ is large in $E(M)$; hence $\operatorname{Im} f=0$ and $E(M)=E(M)_{t} \in \mathscr{T}$. Thus $\mathscr{T}$ is stable. Now choose $M \in \mathscr{F}$; then $E(M) \in \mathscr{F}$ and so the module $E(M) / M$ is torsionfree, and hence projective. Thus $E(M) \cong M \oplus E(M) / M$. This proves that $M$ is injective.

The final statement follows from Lemma 2.1.

THEOREm 2.3. Let $(\mathscr{T}, \mathscr{F})$ be a hereditary torsion theory for ${ }_{R-l l}$ for which cyclic torsionfree modules have projective covers; the following are equivalent:

(1) $\mathscr{F}$ is closed under projective covers, and every torsion module is projective;

(2) $\mathscr{F}$ is closed under homomorphic images, and every torsion module is injective;

(3) $\mathscr{F}$ is closed under homomorphic images, $R=R_{t}+K$ (ring direct sum), and $R_{t}$ is a semi-simple ring with minimum condition.

In addition, whenever (1), (2), and (3) are true, then $\mathscr{T}$ is a TTF class and $(\mathscr{T}, \mathscr{F})$ is centrally splitting.

Proof. (1) $\rightarrow(3)$ : Choose $N \in \mathscr{F}$, and let $L$ be a homomorphic image of $N$. Since $L_{t}$ is projective, there exists a monomorphism $f$ from $L_{t}$ to $N$. But $\operatorname{Hom}\left(L_{t}, N\right)=0$; thus $L_{t}=0$ and $L \in \mathscr{F}$. Thus $\mathscr{F}$ is closed under homomorphic images.

Since $R / R_{t}$ is a cyclic module, it has a projective cover $\pi: P\left(R / R_{t}\right)$ $\rightarrow R / R_{t}$, and $P\left(R / R_{t}\right) \in \mathscr{F}$ by hypothesis. If $n$ denotes the natural epimorphism from $R$ onto $R / R_{t}$, then there exists a homomorphism $f: P\left(R / R_{t}\right) \rightarrow R$ such that $R=\operatorname{Im} f+\operatorname{Ker} n=\operatorname{Im} f+R_{t}$. But $\operatorname{Im} f$ $\in \mathscr{F}$, so that $R_{t} \cap \operatorname{Im} f=0$ and $R=R_{t} \oplus \operatorname{Im} f$. Thus $R=R_{t}+K$ - and we also get the final statement of the theorem - by Lemma 2.1 .

Finally, it is easy to see that $R_{t}$ is a completely reducible ring since every torsion module is projective; this is equivalent to saying that $R_{t}$ is a semi-simple ring with minimum condition.

$(3) \rightarrow(2):$ If $M \in \mathscr{T}$, then $K M=0$ since $\mathscr{F}$ is closed under homomorphic images. Hence $M$ is an injective $R_{t}$-module, and, by Baer's Lemma, it is easy to see that $M$ is an injective $R$-module.

$(2) \rightarrow(1)$ : Let $M \in \mathscr{F}$ have a projective cover $\pi: P(M) \rightarrow M$; then 
$P(M)_{t}$ is injective and $P(M)=P(M)_{t} \oplus P^{\prime}$. Further, $\operatorname{Hom}\left(P(M)_{t}, M\right)$ $=0$ and thus $P(M)_{t} \subseteq \operatorname{Ker} \pi$. Hence $P(M)_{t}$ is small in $P(M)$, and $P(M)=P^{\prime} \in \mathscr{F}$. Thus $\mathscr{F}$ is closed under projective covers.

Since $R_{t}$ is injective, we have $R=R_{t} \oplus K$. Thus, by Lemma 2.1, we have that $R=R_{t}+K$. Since $\mathscr{F}$ is closed under homomorphic images, one can easily see that $M \in \mathscr{T}$ if and only if $K M=0$. But if every $R_{t}$-module is injective, then every $R_{t}$-module is projective. Thus every torsion $R$-module is projective.

3. Stable torsion theories. In [5] the following result is given; its proof depends strongly upon the dualities present in quasi-Frobenius rings.

Theorem B. Let $R$ be a quasi-Frobenius ring and let $(\mathscr{T}, \mathscr{F}$ ) be a hereditary torsion theory for ${ }_{R} \mathscr{C l}$. The following are equivalent:

(1) $\mathscr{T}$ is stable;

(2) $(\mathscr{T}, \mathscr{F})$ is splitting;

(3) $(\mathscr{T}, \mathscr{F})$ is centrally splitting.

It is easily seen that the implications $(3) \rightarrow(2) \rightarrow(1)$ are always true, regardless of the type of ring involved. We are motivated to examine the remaining implications in types of left artinian rings more general that the quasi-Frobenius ones, especially since Fuller [7] has shown that $Q F-3$ rings possess dualities somewhat similar to those in quasi-Frobenius rings.

THEOREM 3.1. Let $R$ be a left artinian generalized uniserial ring, and let $(\mathscr{T}, \mathscr{F})$ be a hereditary torsion theory for ${ }_{R} \mathscr{C l}$. Then $\mathscr{T}$ is stable if and only if $(\mathscr{T}, \mathscr{F})$ is splitting.

Proof. We need only consider the case where $\mathscr{T}$ is stable. Now every module $M$ is a direct sum of indecomposable cyclic submodules, and each of these submodules is a homomorphic image of a left ideal $R e$ where $e$ is a primitive idempotent of $R[10]$. But each such $R e$ has a lattice of submodules which is a finite chain, and thus every homomorphic image of an $R e$ has a lattice of submodules which is a finite chain.

If $L$ is an indecomposable cyclic submodule of $M$, then by the preceding its socle, denoted $\operatorname{soc}(L)$, is simple. Thus either $\operatorname{soc}(L) \in$ $\mathscr{T}$ or $\operatorname{soc}(L) \in \mathscr{F}$. But $\operatorname{soc}(L)$ is large in $L$, so that $L$ is contained in the injective envelope of $\operatorname{soc}(L)$. By hypothesis either $E(\operatorname{soc}(L)) \in$ $\mathscr{T}$ or $E(\operatorname{soc}(L)) \in \mathscr{F}$; thus either $L \in \mathscr{T}$ or $L \in \mathscr{F}$. Hence $M$ splits.

EXAMPLE 3.2. Splitting does not imply centrally splitting in left artinian generalized uniserial rings. 
Let $K$ be a field, and let $R$ be the ring of two by two upper triangular matrices over $K$. Let

$$
I=\left\{\left|\begin{array}{ll}
a & b \\
0 & 0
\end{array}\right| \mid a, b \in K\right\}
$$

then $I$ is an idempotent, two-sided ideal of $R$. Thus by Jans [9],

$$
\mathscr{T}=\left\{M \in_{R} \mathscr{C l} \mid I M=0\right\}
$$

is a TTF class with associated torsion theories $(\mathscr{T}, \mathscr{F})$ and $(\mathscr{C}, \mathscr{T})$, where

$$
\begin{aligned}
\mathscr{F} & =\left\{N \in_{R} \mathscr{C l} \mid \operatorname{Hom}(T, N)=0 \quad \text { for all } T \in \mathscr{T}\right\} \text { and } \\
\mathscr{C} & =\left\{L \in_{R} \mathscr{C l} \mid \operatorname{Hom}(L, T)=0 \text { for all } T \in \mathscr{T}\right\} \\
& =\left\{L \in_{R} \mathscr{C l} \mid I L=L\right\} .
\end{aligned}
$$

Clearly $(\mathscr{C}, \mathscr{T})$ does not split, since $R_{c}=I$ is not a direct summand of $R$. Hence $\mathscr{T}$ is not centrally splitting.

Note that $F(\mathscr{T})=\{I, R\}$; thus for $M \in_{R} \mathscr{C l}, M_{t}=\{x \in M \mid(0: x) \in$ $F(\mathscr{T})\}=\{x \in M \mid I \subseteq(0: x)\}$, where $(0: x)=\{r \in R \mid r x=0\}$. Since $I$ is the only large proper left ideal of $R$, we see that $M_{t}$ is the singular submodule $Z(M)$ of $M$. Also $Z(R)=0$, so that $\mathscr{T}$ is the Goldie and $E(R)$ - torsion class (see [1] and [9] for an explanation of these). It is well-known that the Goldie torsion class is stable; thus $(\mathscr{T}, \mathscr{F})$ splits by Theorem 3.1.

As an aside, we note that the class $\mathscr{C}$ above is hereditary but is not stable. Also we remark that Teply [11, Propositions 4.5 and 4.7] gives several necessary and sufficient conditions for splitting to imply centrally splitting.

Proposition 3.3. Let $R$ be a $Q F-2$ ring, and let $(\mathscr{T}, \mathscr{F})$ be a hereditary torsion theory for ${ }_{R} \mathscr{A l}$. If $\mathscr{T}$ is stable, then every projective module splits.

Proof. If $e$ is a primitive idempotent in $R$, then $\operatorname{soc}(R e)$ is both a simple module and is large in $R e$. Hence $R e$ is contained in the injective envelope of $\operatorname{soc}(R e)$, and thus either $R e \in \mathscr{T}$ or $R e \in \mathscr{F}$. But any projective module $P$ over a left artinian ring $R$ is isomorphic to a direct sum of modules $R e_{\alpha}$, where each $e_{\alpha}$ is a primitive idempotent of $R$. Thus every projective module splits.

If $\mathscr{T}$ is a stable hereditary torsion class for a $Q F-2$ ring, then, by Theorem A and Proposition 3.3, every quasi-injective and every projective module splits. It seems reasonable to conjecture that every module will split, and in fact we have been unable to find examples 
to the contrary.

\section{REFERENCES}

1. J. S. Alin and E. P. Armendariz, TTF-classes and $E(R)$-torsion modules, preprint. 2. J. S. Alin and S. E. Dickson, Goldie's torsion theory and its derived functor, Pacific J. Math., 24 (1968), 195-203.

3. E. P. Armendariz, Quasi-injective modules and stable torsion classes, Pacific J. Math., 31 (1969), 277-280.

4. H. Bass, Finitistic dimension and a homological generalization of semi-primary rings, Trans. Amer. Math. Soc., 95 (1960), 466-488.

5. R. L. Bernhardt, Splitting hereditary torsion theories over semiperfect rings, Proc. Amer. Math. Soc., 22 (1969), 681-687.

6. S. E. Dickson, A torsion theory for abelian categories, Trans. Amer. Math. Soc., 121 (1966), 223-235.

7. K. R. Fuller, The structure of $Q F-3$ rings, Trans. Amer. Math. Soc., 134 (1968), 343-354.

8. P. Gabriel, Des categories abeliennes, Bull. Soc. Math. France, 90 (1962), 323-449.

9. J. P. Jans, Some aspects of torsion, Pacific J. Math., 15 (1965), 1249-1259.

10. T. Nakayama, On Frobeniusean algebras II, Ann. of Math., 42 (1941), 1-22.

11. M. L. Teply, Homological dimension and splitting torsion theories, Pacific J. Math., 34 (1970), 193-206.

12. L. E. T. Wu and J. P. Jans, On quasi-projectives, Ill. J. Math., 11 (1967), 439-448.

Received October 13, 1970 and in revised form April 29, 1971.

The University of North Carolina at Greensboro 


\section{PACIFIC JOURNAL OF MATHEMATICS}

\section{EDITORS}

H. SAMELSON

Stanford University

Stanford, California 94305

C. R. HobBY

University of Washington

Seattle, Washington 98105
J. DugundjI

Department of Mathematics

University of Southern California

Los Angeles, California 90007

RICHARD ARENS

University of California

Los Angeles, California 90024

\section{ASSOCIATE EDITORS}

E. F. BECKENBACH

B. H. NeUMaNN

F. WOLF

K. YoshidA

\section{SUPPORTING INSTITUTIONS}

UNIVERSITY OF BRITISH COLUMBIA

CALIFORNIA INSTITUTE OF TECHNOLOGY

UNIVERSITY OF CALIFORNIA

MONTANA STATE UNIVERSITY

UNIVERSITY OF NEVADA

NEW MEXICO STATE UNIVERSITY

OREGON STATE UNIVERSITY

UNIVERSITY OF OREGON

OSAKA UNIVERSITY
UNIVERSITY OF SOUTHERN CALIFORNIA STANFORD UNIVERSITY

UNIVERSITY OF TOKYO

UNIVERSITY OF UTAH

WASHINGTON STATE UNIVERSITY UNIVERSITY OF WASHINGTON

$\stackrel{*}{*} \stackrel{*}{*} \stackrel{*}{*}$ AMERICAN MATHEMATICAL SOCIETY
NAVAL WEAPONS CENTER 


\section{Pacific Journal of Mathematics}

Vol. 39 , No. 1

May, 1971

Charles A. Akemann, A Gelfand representation theory for $C^{*}$-algebras ....

Sorrell Berman, Spectral theory for a first-order symmetric system of

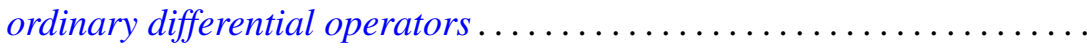

Robert L. Bernhardt, III, On splitting in hereditary torsion theories ........

J. L. Brenner, Geršgorin theorems, regularity theorems, and bounds for determinants of partitioned matrices. II. Some determinantal identities ..........................................

Robert Morgan Brooks, On representing $F^{*}$-algebras .............. 51

Lawrence Gerald Brown, Extensions of topological groups........... 71

Arnold Barry Calica, Reversible homeomorphisms of the real line ........ 79

J. T. Chambers and Shinnosuke Oharu, Semi-groups of local Lipschitzians in

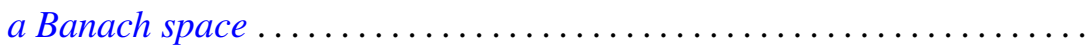

Thomas J. Cheatham, Finite dimensional torsion free rings .............

Byron C. Drachman and David Paul Kraines, A duality between

transpotence elements and Massey products ...................

Richard D. Duncan, Integral representation of excessive functions of a

Markov process ......................................

George A. Elliott, An extension of some results of Takesaki in the reduction

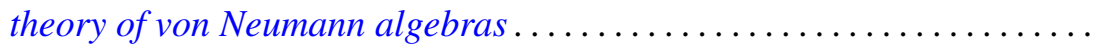

Peter C. Fishburn and Joel Spencer, Directed graphs as unions of partial

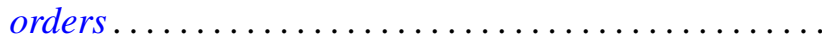

Howard Edwin Gorman, Zero divisors in differential rings ...

Maurice Heins, A note on the Löwner differential equations...

Louis Melvin Herman, Semi-orthogonality in Rickart rings. .

David Jacobson and Kenneth S. Williams, On the solution of linear G.C.D.

equations

Michael Joseph Kallaher, On rank 3 projective planes ... . .

Donald Paul Minassian, On solvable $O^{*}$-groups ...........

Nils Øvrelid, Generators of the maximal ideals of $A(\bar{D})$

Mohan S. Putcha and Julian Weissglass, A semilattice decomposition into

semigroups having at most one idempotent ............

Robert Raphael, Rings of quotients and $\pi$-regularity ....

J. A. Siddiqi, Infinite matrices summing every almost periodic sequence. .

Raymond Earl Smithson, Uniform convergence for multifunctions ...

Thomas Paul Whaley, Mulitplicity type and congruence relations in

universal algebras...

Roger Allen Wiegand, Globalization theorems for locally finitely generated modules... 